\title{
REMARKS CONCERNING THE SUPPORTS OF SOLUTIONS OF PSEUDOPARABOLIC EQUATIONS
}

\author{
WILLIAM RUNDELL AND MICHAEL STECHER
}

\begin{abstract}
This paper deals with the existence of nontrivial solutions of the pseudoparabolic equation $u_{x x t}-u_{t}+u_{x x}=0$ whose supports lie in the half spaces $t>t_{0}, x>x_{0}$. In addition, we show the nonexistence of solutions whose supports lie in bounded strips in $x$ or $t$.
\end{abstract}

I. Introduction. The purpose of this paper is to study the qualitative behavior of solutions of the pseudoparabolic equation

$$
P u \equiv u_{x x t}-u_{t}+u_{x x}=0 .
$$

In particular, we are concerned with the nature of the supports of these solutions.

We shall contrast the results obtained for (1.1) with those of the heat equation

$$
H u \equiv u_{t}-u_{x x}=0 .
$$

One reason for this contrast is that these equations may be used to model similar physical phenomena, with the pseudoparabolic equation corresponding to a higher order correction to the model that gives rise to the heat equation. Examples of this may be found in [1], [2] and [6]. In many cases the same boundary value problems are well posed for both these equations [7], [8], and the solution of the heat equation may be approximated by solutions of the pseudoparabolic equation.

In §II we discuss the following question. Can a nontrivial solution of either (1.1) or (1.2) possess the property: for some $x_{0}$ the solution vanishes identically in $x$ and $t$ for $x<x_{0}$ ? For the heat equation this is impossible, since, as is well known, every solution of this equation is analytic in $x$, cf. [5]. We shall show however that such behavior is possible for solutions of pseudoparabolic equations. This leads to a further question: Can equation (1.1) have a solution which has compact support in $x$ ? Surprisingly this is not possible.

The third section of this paper deals with the corresponding question for $t$, that is, for some $t_{0}$ can a solution of either (1.1) or (1.2) be such that it vanishes identically in $x$ and $t$ for $t<t_{0}$, but not for $t>t_{0}$ ? That such behavior is possible for solutions of the heat equation is a classical result due to Tychonov [4]. We show that this is also possible for a solution of $P u=0$.

Received by the editors August 2, 1976.

AMS (MOS) subject classifications (1970). Primary 35A20, 35B99; Secondary 35K05.

(C) American Mathematical Society 1977 
Again the question arises: Is it possible to have a solution with compact support in $t$ ? Tychonov's method can be used to show that this may happen for solutions of the heat equation. For the pseudoparabolic case this is not possible.

In the final section, we discuss extensions of these results to equations in more than one space variable and to nonconstant coefficients.

Throughout this paper by solutions we mean strong solutions. That is, solutions that are twice continuously differentiable with respect to $x$ and continuously differentiable with respect to $t$.

II. Solutions with support contained in the half space $x>x_{0}$. We shall first obtain a representation due to Colton [3] for solutions of pseudoparabolic equations in one space variable. For convenience we outline his method.

We define the adjoint equation of $P u=0$ to be

$$
P^{*} v \equiv v_{x x t}+v_{t}-v_{x x}=0 .
$$

It is shown in [3] that there exists a unique solution of (2.1) in the rectangle $R$ which is bounded by the lines $x=0, x=\xi, t=0, t=\tau$ and satisfies the boundary conditions

$$
v(\xi, t, \xi, \tau)=0, \quad v(x, \tau, \xi, \tau)=0, \quad v_{x}(\xi, t, \xi, \tau)=1-e^{(t-\tau)} .
$$

Now integrate the identity

$$
v_{t} P u-u_{t} P^{*} v=\frac{\partial}{\partial x}\left\{u_{x t} v_{t}-u_{t} v_{x t}+u_{x} v_{t}+u_{t} v_{x}\right\}-\frac{\partial}{\partial t}\left\{u_{x} v_{x}\right\}
$$

over the rectangle $R$ to obtain, by Green's theorem,

$$
\begin{aligned}
\int_{0}^{\tau} \int_{0}^{\xi}\left[v_{t} P u-u_{t} P^{*} v\right] d x d t= & \int_{\partial R}\left(u_{x t} v_{t}-u_{t} v_{x t}+u_{x} v_{t}+u_{t} v_{x}\right) d t \\
& +\int_{\partial R} u_{x} v_{x} d x
\end{aligned}
$$

Using conditions (2.2) we derive the representation

$$
\begin{aligned}
u(\xi, t)= & h(\xi)-\int_{0}^{\xi} h^{\prime}(x) v_{x}(x, 0, \xi, \tau) d x \\
& +\int_{0}^{\tau}\left[g^{\prime}(t) v_{t}(0, t, \xi, \tau)-f^{\prime}(t) v_{x t}(0, t, \xi, \tau)\right. \\
& \left.+g(t) v_{t}(0, t, \xi, \tau)+f^{\prime}(t) v_{x}(0, t, \xi, \tau)\right] d t
\end{aligned}
$$

where

$$
u(0, t)=f(t), \quad u_{x}(0, t)=g(t), \quad u(x, 0)=h(x) .
$$

It is assumed that $f, g \in C^{1}[0, \tau]$ and $h \in C^{2}[0, \xi]$. It can be shown that (2.5) represents the unique solution to $P u=0$ which satisfies the boundary conditions (2.6).

Our first result now follows rather easily. Choose $x_{0}>0$ and $f(t)=g(t)=$ 0 . Choose $h(x) \in C^{2}(\mathbf{R})$ to have support contained in $x>x_{0}$. From (2.5) it is 
clear that we obtain a solution $u(\xi, \tau)$ such that $u(\xi, \tau)=0$ for $0 \leqslant \xi<x_{0}$, but does not vanish identically. From (2.5) it is clear that this solution may be extended by zero for $\xi \leqslant 0$.

We remark that this difference in support properties of the heat equation and pseudoparabolic equation can perhaps best be explained by noting that the heat operator is "infinitely smoothing" on the initial data whereas the pseudoparabolic "preserves the smoothness" of the initial data.

We now prove that there are no solutions of compact support in $x$.

TheOREM 1. Let $u(x, t)$ be a solution of $P u=0$ such that $u(x, t)=0$ for $x<x_{0}$ and $x>x_{1}$ with $x_{0}<x_{1}$, then $u(x, t)$ vanishes identically.

Proof. Without loss of generality assume $x_{0}=0$ and $x_{1}=\pi$. Since we are assuming that our solutions are strong solutions we have that $u(x, 0) \in$ $C^{2}(\mathbf{R})$. This is a consequence of the fact that the initial boundary value problem for pseudoparabolic equations is well posed both backwards and forwards in time and preserves the smoothness of the initial data.

We point out that $u_{x}(0, t)=0$.

By separation of variables we may represent a solution, for $0 \leqslant x \leqslant \pi$, by

$$
u(x, t)=\sum_{n=1}^{\infty} a_{n} e^{-\lambda_{n} t} \sin n x, \quad \lambda_{n}=\frac{n^{2}}{1+n^{2}},
$$

where

$$
u(x, 0)=\sum_{n=1}^{\infty} a_{n} \sin n x
$$

and from our assumption on $u(x, 0)$ we have $\left\{n a_{n}\right\}_{n=1}^{\infty} \in l^{1}$. Also

$$
0=u_{x}(0, t)=\sum_{n=1}^{\infty} n a_{n} e^{-\lambda_{n} t}
$$

It is clear that (2.9) converges uniformly in $t$. We then take the Laplace transform $L(t \rightarrow s)$ to obtain for $s \geqslant 0$,

$$
0=\sum_{n=1}^{\infty} n a_{n}\left(s+\lambda_{n}\right)
$$

The series $f(z)=\sum_{n=1}^{\infty} n a_{n}\left(z+\lambda_{n}\right)$ represents a meromorphic function of $z$ with poles at $z=-\lambda_{n}, n=1,2, \ldots$, and such that $f(z)$ is zero for $z$ real and positive. Hence $f(z)$ is identically zero and the residues at the poles are also zero and, thus, $a_{n}=0$ for all $n$. It follows that $u(x, t)=0$ for $0<x<\pi$.

We have, in fact, proved a slightly stronger result, namely that if $u(x, t)=$ 0 for $x<x_{0}$ and $u\left(x_{1}, t\right)=0$ for some $x_{1}>x_{0}$ then $u(x, t)=0$ for $x \leqslant x_{1}$.

III. Solutions with support contained in the half space $t<t_{0}$. We now show the existence of nontrivial solutions of $P u=0$ that vanish identically for $t<t_{0}$.

The method which Tychonov used to construct solutions of this type for the heat equation is not applicable to the pseudoparabolic equation. It is 
possible, however, by the use of integral operator techniques to show the existence of such solutions.

In [7] it is shown that every solution $u(x, t)$ of $P u=0$ with $u(x, 0)=0$ may be written in the form

$$
u(x, t)=h(x, t)+\int_{0}^{t} \int_{0}^{1} K(x, \sigma, t-\tau) h_{\tau}\left(x \sigma^{2}, \tau\right) d \sigma d \tau,
$$

where $h(x, t)$ satisfies $h_{x x t}=0$ with $h(x, 0)=0$, that is,

$$
h(x, t)=x g_{1}(t)+g_{2}(t), \quad g_{1}(0)=g_{2}(0)=0,
$$

and

$$
K(x, \sigma, t)=\sum_{n=1}^{\infty}\left(\frac{x}{2}\right)^{2 n} \frac{\left(1-\sigma^{2}\right)^{n-1}}{n !(n+1) !} a_{n}(t)
$$

with

$$
a_{n+1}^{\prime}(t)=a_{n}^{\prime}(t)-a_{n}(t), \quad a_{n}(0)=1, \quad a_{1}(t)=1+t .
$$

Note that $a_{n}(t)$ is a polynomial of degree $n$ in $t$. Clearly it suffices to choose $\tau_{0} \geqslant 0$. By choosing for example $g_{1}(t)=0$ and $g_{2}(t)$ to be continuously differentiable with support in $t>t_{0}$, the existence of the required solution to $P u=0$ follows from the representation (3.1).

We now use this representation to show that solutions of $P u=0$ with compact support in time must be identically zero. In fact we prove a stronger result.

THEOREM 2. If for some $t_{0}$ and $t_{1}$ we have $u\left(x, t_{0}\right)=u\left(x, t_{1}\right)=0$, then $u(x, t)=0$ for $t_{0} \leqslant t \leqslant t_{1}$.

Proof. Without loss of generality choose $t_{0}=0$. From (3.1) we have,

$$
0=x g_{1}\left(t_{1}\right)+g_{2}\left(t_{1}\right)+\int_{0}^{t_{1}} \int_{0}^{1} K\left(x, \sigma, t_{1}-\tau\right)\left[\sigma^{2} g_{1}^{\prime}(\tau)+g_{2}^{\prime}(\tau)\right] d \sigma d \tau
$$

Replacing $K$ by its power series, and equating powers of $x$ we obtain

$$
\begin{gathered}
g_{1}\left(t_{1}\right)=g_{2}\left(t_{1}\right)=0 \\
\int_{0}^{t_{1}} a_{n}\left(t_{1}-\tau\right) g_{i}^{\prime}(\tau) d \tau=0, \quad i=1,2 .
\end{gathered}
$$

From (3.2) and (3.7) we have that $g_{i}^{\prime}(\tau)$ is orthogonal (in $L^{2}\left[0, t_{1}\right]$ ) to 1 . From (3.8) and the form of the $a_{n}$ 's we have that $g_{i}^{\prime}(\tau)$ is orthogonal to $t^{n}$ for $n=1,2, \ldots$ Hence $g_{i}(\tau)$ is a constant and thus 0 .

We remark that in Tychonov's example for the heat equation, the solution $u(x, t)$ grows faster than $e^{a x^{2}}$ for any constant $a$, that is, the solution does not lie in the uniqueness class for the heat equation. In the case of the pseudoparabolic equation, $u(x, t)$ does not lie in $L^{2}(\mathbf{R})$, a sufficient condition for a unique solution to the Cauchy problem for this equation. 
IV. Generalizations. We conclude by briefly indicating possible extensions of these results to nonconstant coefficients and to more than one space variable.

In §II Colton's representation is valid only for one space variable, but may be extended to variable even time dependent coefficients [3]. Thus our results can be extended to this case. The fact that there are no nontrivial solutions of compact support in $x$ has an obvious analogue, by generalised Fourier series, to $n$ space variables with time independent coefficients.

In §III the integral operator may be extended to $n$ variables where $h(x, t)$ satisfies $\Delta h_{t}=0$. The kernel $K$ has $x$ replaced by

$$
r=\sqrt{ }\left(x_{1}^{2}+x_{2}^{2}+\cdots+x_{n}^{2}\right) \text {. }
$$

A similar representation is also possible when the coefficients depend analytically on $r^{2}$ [7]. In this case $K(r, \sigma, t)$ has the form

$$
K(r, \sigma, t)=\sum_{k=1}^{\infty} e^{(k)}\left(r^{2}, t\right)\left(1-\sigma^{2}\right)^{k-1}
$$

where $e^{(k)}\left(r^{2}, t\right)$ is analytic in $r^{2}$ and $t$ and for fixed $r^{2}$ is a polynomial of degree $k$ in $t$. The existence of solutions with support in the half space $t>t_{0}$ can now be extended to $n$ space variables and to pseudoparabolic equations with coefficients which are analytic functions of $r^{2}$. We have been unable to extend Theorem 2 to more than one space variable. However in this case when the coefficients are analytic functions of $x^{2}$, the same proof goes over as before, by equating even and odd functions of $x$.

Theorem 2 is, in general, false when the coefficients depend on $t$, for example

$$
u(x, t)=t(1-t) e^{t} \sinh x
$$

is a solution of the equation

$$
u_{x x t}-\left(t^{2}-t+1\right) u_{t}+\left(t^{2}+t-1\right) u_{x x}=0 .
$$

\section{REFERENCES}

1. G. Barenblat, I. Zheltov and I. Kochiva, Basic concepts in the theory of seepage of homogeneous liquids in fissured rocks, J. Appl. Math. Mech. 24 (1960), 1286-1303.

2. P. J. Chen and M. E. Gurtin, On a theory of heat conduction involving two temperatures, Z. Angew. Math. Phys. 19 (1968), 614-627.

3. D. L. Colton, Pseudoparabolic equations in one space variable, J. Differential Equations, 12 (1972), 559-565. MR 48 \#476.

4. G. Hellwig, Partial differential equations, Blaisdell, Waltham, Mass., 1964.

5. L. Hörmander, Linear partial differential operators, Springer-Verlag, Berlin and New York, 1969. MR $40 \# 1687$.

6. E. H. Milne, The diffusion of imprisoned radiation through a gas, J. London Math. Soc. 1 (1926), 40-51.

7. W. Rundell and M. Stecher, A method of ascent for parabolic and pseudoparabolic partial differential equations, SIAM J. Math. Anal. 7 (1976), 898-912.

8. R. E. Showalter and T. W. Ting, Pseudoparabolic partial differential equations, SIAM J. Math Anal. 1 (1970), 1-26.

Department of Mathematics, Texas A\&M University, College Station, Texas 77840 